\title{
Aplicación de criterios de búsqueda y selección de textos para la elaboración de trabajos académicos en estudiantes universitarios: un estudio de caso
}

\author{
Application of search criteria and text selection for the \\ elaboration of academic works in university students: a case study
}

Guisella Ivonne Azcona Avalos*

Universidad Tecnológica del Perú, Lima, Perú

ORCID: https://orcid.org/0000-0002-5476-6699

Janina del Rocío Navarro Linares

Universidad Tecnológica del Perú, Lima, Perú

ORCID: https://orcid.org/0000-0002-8998-2607

*Correspondencia:

Email: ahombrosdegigantes@utp.edu.pe
Citar como:

Azcona, G. \& Navarro, J. (2021). Aplicación de criterios de búsqueda y selección de textos para la elaboración de trabajos académicos en estudiantes universitarios: un estudio de caso. Propósitos y Representaciones, 9(3), e825. http://dx.doi.org/10.20511/pyr2021.v9n3.825 


\section{Resumen}

La presente investigación muestra las dificultades que presentan los estudiantes para la aplicación de criterios de búsqueda y selección de textos para la elaboración de trabajos académicos en el primer año universitario. A partir de ello, se presentó una propuesta de mejora que consistió en tres talleres didácticos en tres grupos de la modalidad presencial y en tres grupos de la modalidad semipresencial de la Universidad Tecnológica del Perú, ejecutados en el segundo semestre del año 2019. Asimismo, se trabajó según el paradigma interpretativo, enfoque cualitativo, diseño descriptivo y método inductivo. Se demostró que tanto en la modalidad presencial y semipresencial hubo efectos diversos en la permanencia de estudios, asimilación del aprendizaje y usos de los recursos de información tecnológica para investigación académica.

Palabras clave: Fuentes de información; Criterios de selección; Técnicas de investigación; Investigación sobre literatura científica; Aprendizaje en grupo.

\section{Summary}

The present investigation shows the difficulties that students present for the application of search criteria and selection of texts for the preparation of academic works in the first year of university. Based on this, an improvement proposal was presented that consisted of three didactic workshops in three groups of the face-to-face modality and in three groups of the blended modality of the Technological University of Peru, execut-ed in the second semester of 2019. Likewise, we worked according to the interpretive paradigm, qualitative approach, descriptive design and inductive method. It is concluded that there were diverse effects both in the face-to-face and blended modality, in the permanence of studies, in the assimilation of learning and in the uses of technological information resources for academic research.

Keywords: Information sources; Selection criteria; Research techniques; Research on scientific literature; Group learning.

\section{Introducción}

Una de las características usuales dentro de la vida universitaria es la dificultad que presentan los estudiantes en la búsqueda y selección de fuentes bibliográficas que permitan elaborar sus trabajos académicos. Las universidades presentan estrategias educativas que exigen a los docentes realizar un seguimiento exhaustivo y permanente a los trabajos de investigación de los estudiantes. Tal como lo indica Perdomo (2008), es necesario que se tenga mayor conciencia al escoger los contenidos oportunos para investigar (p. 115). Esto genera, a su vez, ciertas relaciones con la deficiencia de com-prensión lectora y la escritura académica.

El proceso de un trabajo de investigación requiere de un dominio estratégico de la información a tratar, que implica no partir solo de intuiciones sino de competencias metodológicas. En ese sentido, Negrete (2002) alertó que, en la actualidad, seleccionar información requiere de criterios apropiados sobre todo en los recursos digitales (p. 55). Sin una adecuada conciencia del proceder estratégico, el estudiante rápidamente puede desinteresarse o enfrentar un impacto negativo que condicione su experiencia como investigador.

Perdomo (2008) manifiesta que el desconocimiento de estrategias adecuadas hace que sea infructuoso las búsquedas en línea (p. 109). Teniendo presente dicha problemática, los docentes podrán involucrar al estudiante a que tome conciencia de que la única manera de aprender a investigar es investigando; es decir, planteando modos de cómo buscar soluciones a problemas iniciales de investigación. Es por ello que, al partir del enfoque cualitativo en esta investigación, se extrae información que permita establecer si existe tal relación problemática, 
cuáles son los criterios iniciales de cómo investigan y después cómo realizan dicha selección con orientación, para verificar si es un problema de estrategia o de falta de adaptación a una nueva cultura académica (Carlino, 2003, p. 17). De este modo, se quiere establecer los criterios convenientes que permi-tan tanto a los estudiantes como a los docentes considerar para un óptimo desarrollo y sincero interés por el trabajo académico universitario.

En cuanto a las acciones educativas innovadoras, la investigación busca insertarse con el modelo pedagógico por competencias, ya que es el modelo establecido por la Universidad Tecnológica del Perú (UTP) para el logro óptimo del desempeño de conocimientos y habilidades para el estudiante de pregrado. Con el objetivo de realizar nuestra investigación, diagnosticaremos la problemática a partir de la técnica de la observación que permite determinar el grado de dominio del estudiante según su forma de desenvolverse en un determinado contexto y también posibilita el registrar su nivel de dificultad sobre un determinado aprendizaje (Moreno, 2012, p. 9). Mediante ello, se re-gistrarán los criterios que ha considerado para buscar y seleccionar textos con el fin de delimitar su tema de investigación.

Por tanto, el desarrollo de esta investigación contribuye al diagnóstico y descripción de los hábitos de los estudiantes en el proceso de búsqueda y selección de fuentes para la elaboración de trabajos académicos. Con ello, hace posible identificar qué criterios consideran como adecuados para tal fin y si hay alguna relación con la deficiencia de comprensión de lectura académica. A partir de ello, se desea generar una propuesta de mejora que implique un compromiso significativo con la investigación académica, y así incrementar las oportunidades de innovar, analizar y contribuir en el mejoramiento de la calidad educativa. De poder desarrollar dicha intervención, en el futuro, los jóvenes de primer año podrán generar planteamientos de investigación más eficientes en sus res-pectivos campos.

\section{Método}

El paradigma desarrollado ha sido interpretativo. Según Ricoy (2006) dicho modelo de estilo cualitativo se refiere a la creación global que respeta el carácter contextual de la investigación utilizando diversas técnicas que permite una flexibilidad en las observaciones y discusiones dentro de la investigación para generar soluciones adecuadas. (p. 9)

Por otro lado, el diseño de este trabajo corresponde a investigación-acción. Se consideró este diseño puesto que el objetivo es generar cambios a través de la intervención activa durante el proceso de la investigación (Hernández \& Mendoza, 2018, p. 552). Asimismo, el método de investigación es inductivo, ya que se utiliza la observación y el análisis para registrar las causas de los fenómenos estudiados (Pimienta \& De la Orden, 2017, p. 61).

En cuanto al escenario de estudio, Taylor \& Bogdan (1987) argumentaron que el escenario ideal es aquel donde el observador tiene a su disposición los aspectos que siguen: (a) fácil acceso a la información, (b) el contexto propicio para el proceso de investigación, (c) una buena relación con los participantes y (d) la posibilidad de recoger datos sin ningún tipo de dificultad. En lo que respecta a la presente investigación, la comunidad universitaria de la UTP Lima Centro cumple con todo lo antes mencionado, y los participantes del estudio (los estudiantes de modalidad presencial y modalidad semipresencial) son conocedores de la investigación, de la cual colaboran a través del diagnóstico y los grupos focales durante todo el proceso de investigación. De esta forma, todos los datos recabados tendrán como objetivo comprender la realidad y solucionar la problemática hallada.

Dentro de la comunidad universitaria, se trabajaron con las 2 modalidades académicas: modalidad presencial y modalidad semipresencial (CGT). En cuanto a la modalidad presencial, se trabajó con la sección 5273, con los grupos siguientes: Las Pun, Los Inquisidores, Los Bakyardigans y Los Panchis; mientras que la modalidad CGT, se traba-jó con las secciones 18400 
(grupo 1, grupo 2, grupo 3) y 18402 (grupo 1, grupo 2, grupo 3). En la Tabla 1, se presenta la síntesis de lo antes expuesto.

Tabla 1.

Codificación de los sujetos participantes en la investigación

\begin{tabular}{lll}
\hline Sujeto & Letra & Código \\
\hline $\begin{array}{l}\text { Grupos de enfoque: modalidad } \\
\text { presencial }\end{array}$ & GP & GP1, GP2, GP3, GP4 \\
$\begin{array}{ll}\text { Grupos de enfoque: modalidad CGT } & \text { GCGT }\end{array}$ & $\begin{array}{l}\text { GCGT1, GCGT2, GCGT3, GCGT4, } \\
\text { GCGT5, GCGT6 }\end{array}$ \\
\hline
\end{tabular}

Fuente: Elaboración propia.

Las técnicas de recolección, según Hernández y Mendoza (2018), son aquellas pautas que dirigen a los investigadores en cada etapa del proceso de investigación, permitiendo el recojo de datos acorde a la intencionalidad de la investigación. En función a ello, para esta investigación se trabajará con las siguientes técnicas: observación y focus group. En cuanto a la observación, los autores antes mencionados, señalan que el observador cualitativo usa todos los elementos sensitivos que tiene a su alcance, desarrolla capacidad minuciosa de atención, así como de análisis conductuales, y posee capacidad de reflexión y flexibilidad (p. 449). En cuanto al focus group, afirman que la perspectiva e interacción de cada uno de los miembros de los grupos focales es de importancia para el investigador (p. 455).

En cuanto al instrumento, Hernández y Mendoza (2018) argumentaron que su finalidad es ordenar el contenido de la información que se ha logrado obtener sin dejar de lado ningún tipo de información. La observación y el focus group permiten, por ello, retroalimentar y perfeccionar la información durante y después del proceso de la investigación.

En lo que respecta a esta investigación, se realizó a través de los siguientes instrumentos aplicados con sus respectivos materiales de registro presentados (ver Tabla 2).

Tabla 2.

Cuadro de técnicas, instrumentos y material de registro

\begin{tabular}{lll}
\hline Técnicas & Instrumentos & Material de registro \\
\hline Observación & Lista de cotejo & $\begin{array}{l}\text { Registro de asistencia } \\
\text { Material de trabajo grupal (por } \\
\text { taller) }\end{array}$ \\
\hline
\end{tabular}

Fuente: Elaboración propia.

El primer taller estuvo dirigido a la selección y búsqueda de información (taller 1). Se ha desarrollado en tres momentos:

1. Explicación teórica de la búsqueda y selección de información, así como la muestra detallada de los buscadores académicos tanto nacionales como internacionales.

2. Uso correcto de los operadores booleanos y del tesauro (UNESCO) para una mejor búsqueda y selección de información.

3. Aplicación de lo aprendido a través de una actividad en físico y la actividad metacognitiva a través del Kahoot. Lo descrito anteriormente es de modo grupal.

El segundo taller estuvo dirigido a la clasificación de fuentes de información, la selección y búsqueda de información (taller 2). Se ha desarrollado en tres momentos: 
1. Explicación del uso correcto de la técnica de las fichas para clasificar la información seleccionada. Hay de dos formas: textual y parafraseada.

2. Se entrega fichas de cartulina de colores (azul para las textuales, rosadas para las parafraseadas) para que puedan catalogarlas y escribir la información seleccionada. Esta parte es manuscrita; luego, se les solicitará que sean tipeadas para su uso en la redacción del artículo académico.

3. Con la ayuda del esquema de redacción, se ordenan las fichas: aquellas que serán usadas para redactar la introducción y las que serán redactadas en el cuerpo, de acuerdo con cada argumento. Finaliza con la metacognición a través del Kahoot.

El tercer taller estuvo dirigido al estilo de citación APA y herramientas de apoyo para la investigación (taller 3). Se ha desarrollado en tres momentos:

1. La parte teórica.

2. La parte práctica (en la sala de cómputo).

3. La metacognición (Kahoot).

Este último taller fue ejecutado por una especialista en corrección de estilo, la profesora Patricia Vilcapuma Vinces.

En lo que respecta a las categorías y subcategorías, son elementos usados en la investigación que nos permiten delimitar los criterios tratados de manera sistemática, estructurada y de fácil identificación. En la Tabla 3 y Tabla 4, se presentan las matrices de las categorías y subcategorías desarrolladas durante el presente proyecto:

\section{Tabla 3.}

Matriz categorización criterios de búsqueda de textos

\begin{tabular}{ll}
\hline Categoría & Subcategorías \\
\hline & Barra de direcciones \\
Criterios de búsqueda de textos & Motores de búsqueda \\
& Directorios \\
\hline
\end{tabular}

Fuente: Elaboración propia.

Tabla 4.

Matriz categorización criterios de selección de textos

\begin{tabular}{ll}
\hline Categoría & Subcategorías \\
\hline & Contenido \\
& Autoría \\
Criterios de selección de textos & Actualidad \\
& Facilidad de uso \\
& Presentación \\
& Interactividad \\
& Pertinencia \\
\hline
\end{tabular}

Fuente: Elaboración propia.

En cuanto al cumplimiento del rigor científico, durante el desarrollo del proyecto en las aulas seleccionadas, tanto en la modalidad presencial como en la semipresencial, y, para mantener la eficacia de la investigación, se ha trabajado en base a lo estipulado por la universidad, a través del uso de sus manuales de citas y referencias APA (American Psycological Association).

Con respecto a la dependencia, Hernández, Fernández y Baptista (2014) argumentaron que es una especie de confiabilidad cualitativa, esto basándose en diversos autores: citando a 
Guba y Lincoln (1989), la dependencia es denominada consistencia lógica, mientras que, citando a Mertens (2010), equivale más bien al concepto de estabilidad. Por consiguiente, el grado de la dependencia permite el análisis objetivo e imparcial con el tipo de información para que pueda ser aplicada en contextos similares. Es, como señalaría Creswell (2013), la consistencia de los resultados (citado por Hernández, Fernández y Baptista, 2014). Para Hernández y Mendoza (2008), citados por Hernández, Fernández y Baptista (2014), argumentaron que los datos deben ser debidamente registrados y constantemente contrastados por distintos investigadores buscando una congruencia unitaria.

En cuanto a la credibilidad, según Saumure y Given (2008), es “máxima validez” y, de acuerdo con The SAGE Glossary of the Social and Behavioral Sciences (2009), es cuando el investigador (en este caso, investigadoras) ha logrado asimilar el significado exhaustivo de las experiencias de los participantes (ambos citados por Hernández, Fernández y Baptista, 2014); en especial, referidos al planteamiento del problema y es capaz de expresar con claridad y precisión lo que han intentado comunicar los participantes. Por otro lado, Mertens (2010) la definió como la correspondencia entre la percepción del participante sobre la investigación planteada y la capacidad del investigador por entender el mensaje (citado por Hernández, Fernández y Baptista, 2014).

Con respecto a la transferencia (aplicabilidad de resultados), para Hernández, Fernández y Baptista (2014), citando a Savin-Baden y Major (2013), Morse (2012) y Williams et al. (2005), se trata de la condición de extrapolar ciertas características de los resultados a otros contextos.

Por último, en cuanto a la confirmación o confirmabilidad, Hernández, Fernández y Baptista (2014) la explican, citando a Mertens (2010) y Guba y Lincoln (1989), como la condición de indagar e interpretar los datos en su fuente, que implica trabajo de campo, comparación, seguimiento a los participantes y reflexión por parte del investigador (en este caso, de ambas investigadoras).

Según Hernández y Mendoza (2018), el sistema de análisis de datos en las investigaciones de enfoque cualitativo depende del objeto de estudio. Por ende, el método que se utilizará será el interpretativo. En la Tabla 5, se explica lo mencionado.

Tabla 5.

Secuencia del análisis de datos del estudio

\begin{tabular}{|c|c|c|c|}
\hline Temática & Procesos & Elaboración de resultados & Informe de hallazgos \\
\hline $\begin{array}{l}\text { Transcripción de datos en } \\
\text { matriz por cada aspecto } \\
\text { de análisis. }\end{array}$ & $\begin{array}{l}\text { Interpretación } \\
\text { Reducción } \\
\text { Codificación } \\
\text { Categorización }\end{array}$ & $\begin{array}{l}\text { Presentación de tendencias no } \\
\text { esperadas. } \\
\text { Presentación de tendencia } \\
\text { esperadas. } \\
\text { Discusión crítica. } \\
\text { Conclusiones previas. }\end{array}$ & $\begin{array}{l}\text { Presentación de } \\
\text { resultados según } \\
\text { objetivos del estudio. }\end{array}$ \\
\hline
\end{tabular}

Fuente: Elaboración propia.

En relación a los aspectos éticos, siguiendo a Hernández, Fernández y Baptista (2014) y Hernández y Mendoza (2018), lo realizado en la presente investigación está bajo los procedimientos teóricos y metodológicos que ayudan en la construcción de una información desde la perspectiva propia. De igual forma, está avalada por diversos autores que han tratado el tema y de los participantes que han colaborado con sus intervenciones, reservándonos la identidad de cada uno de ellos. 


\section{Resultados}

En los focus group realizados a los estudiantes de ambas modalidades (presencial y CGT) sobre la aplicación de criterios de búsqueda y selección de textos para la elaboración de trabajos académicos en estudiantes de I ciclo del curso de Investigación Académica de la Universidad Tecnológica del Perú, los resultados interpretativos se exponen a continuación.

En relación con el objetivo general "Describir qué criterios habituales usan los estudiantes para la búsqueda y selección de textos en la elaboración de trabajos académicos en el curso de Investigación académica de la Universidad Tecnológica del Perú", se logra evidenciar que pocos estudiantes, tanto de la modalidad presencial como de CGT, tienen conocimientos previos de búsqueda de información. Sin embargo, no los adecua-dos (Google buscador y Wikipedia, por ejemplo). Con respecto a selección de textos, sus conocimientos se restringen a la técnica del subrayado y fichaje textual. La mayoría no tiene conocimientos adecuados sobre búsqueda y selección de información.

En cuanto al primer objetivo específico "Identificar los criterios de búsqueda de textos que los estudiantes asumen como adecuados para la elaboración de trabajos académicos en el curso de Investigación académica de la Universidad Tecnológica del Perú", son cuatro los criterios de búsqueda de textos que los estudiantes de la modalidad presencial han logrado asumir como adecuados luego de las experiencias de los talleres aplicados. En primer lugar, resaltan los buscadores académicos (Scopus, Scielo, Redalyc, Dialnet, etc.); ya que, gracias a ellos, pudieron encontrar información confiable que les serviría para la realización de su investigación. En segundo lugar, están los operadores booleanos, pues les facilitaba el modo de búsqueda y la interacción con información más precisa. En tercer lugar, la búsqueda de información (científica) se basa no solo en los textos encontrados en los buscadores académicos; también en los repositorios (como ALICIA y RENATI), donde se almacenan proyectos de investigación, tesis, libros, entre otros. Finalmente, estuvo presente la seguridad de textos confiables, la lectura de textos adecuados, referencias, libros y artículos; puesto que, a través de la experiencia del primer taller, pudieron interactuar con información confiable, que tiene el respaldo de diferentes casas editoras, revistas científicas y organizaciones de investigación científica.

Mientras que, en el mismo objetivo, son tres los criterios de búsqueda de textos que los estudiantes de la modalidad semipresencial (CGT) han logrado asumir como ade-cuados luego de las experiencias de los talleres aplicados. En primer lugar, identificaron la importancia de los repositorios académicos porque permiten un respaldo objetivo de información fidedigna, así como la necesidad de una selección confiable de documentos académicos desde la selección de expertos. En segundo lugar, destacó la necesidad de organización de tiempo y de trabajo colaborativo para revisar los documentos, debido a que todos trabajan y estudian. Finalmente, la capacidad de sesgar los documentos confiables y académicos de aquellos que no lo son.

Con respecto al segundo objetivo específico "Identificar los criterios de selección de textos que los estudiantes asumen como adecuados para la elaboración de trabajos académicos en el curso de Investigación académica de la Universidad Tecnológica del Perú", los estudiantes de la modalidad presencial identificaron dichos criterios luego de la experiencia de los talleres aplicados. En primer lugar, se presentó el tema del antiplagio, ya que, gracias a los talleres, se logra apreciar cuán importante es la responsabilidad intelectual al citar y referenciar los textos por usar para la investigación que se realizará. En segundo lugar, se consideró el uso de las TICs y sala de cómputo, debido a la experiencia del uso de las computadoras y no el tener la necesidad de trasladar sus laptops o tabletas a las clases de investigación académica; además, se pudo trabajar en tiempo real tanto los ejercicios como el propio trabajo académico en clase. En tercer lugar y en consecuencia de lo anterior, entra el monitoreo, ya que el docente puede monitorear el avance del trabajo de investigación, los ejercicios y las dudas con respec-to a su investigación en 
tiempo real. En cuarto lugar, estuvo presente el parafraseo (explicar con sus propias palabras lo que el autor o autores quisieron decir en su texto) con el fin de evitar la deshonestidad académica e intelectual dentro del proceso de investigación. En quinto lugar, se usó y aplicó el estilo APA en los trabajos de investigación; de esa forma, de manera ordenada y honesta, presentarán los trabajos de investigación. Por último, los estudiantes manifestaron que debe mejorar el wifi para trabajar dentro del proceso de investigación, capacitar a los docentes en estilo APA o ser acompañados por un especialista, trabajar más en la técnica de fichaje para poder seleccionar mejor la información leída y, ya dentro del proceso de redacción del artículo académico, usar la sala de cómputo y así evitar algún tipo de riesgo con su equipo electrónico (robo).

Mientras que, en el mismo objetivo, se identificó los criterios de selección de textos que los estudiantes de la modalidad semipresencial (CGT) asumen como adecuados luego de la experiencia de los talleres aplicados. En primer lugar, los estudiantes reconocieron la utilidad de la organización de ideas a través del fichaje; no solo como técnica de redacción sino también como técnica de estudio para la lectura y escritura académica. Asimismo, reconocieron la importancia del fichaje no solo para el curso de Investigación Académica, sino para la práctica educativa en general. En segundo lugar, con respecto al estilo APA, los estudiantes que participaron estuvieron de acuerdo en recibir reforzamiento adicional a través de talleres, porque en ellos reforzaron lo aprendido de manera más especializada, detallada y profunda. Les permitió discernir no solo para la escritura académica, sino también para saber analizar y evaluar lecturas académicas. En tercer lugar, aquellos que no tuvieron la oportunidad de participar en los talleres APA recomendaron la necesidad de un reforzamiento adicional, porque lograron compararse con aquellos que sí participaron en los talleres. Finalmente, en cuarto lugar, la expectativa del uso del fichaje y la aplicación del estilo APA para recolección y redacción de información se asumen como fundamentales para su práctica académica, en sus carreras y en sus trabajos. Incluso, destacaron la necesidad de una práctica constante no solo de manera digital, sino de escritura manual para fichaje y luego digital para trabajos académicos.

En la Figura 1, se presenta el resultado conclusivo del proceso de triangulación, donde se presentan las categorías emergentes de la investigación.

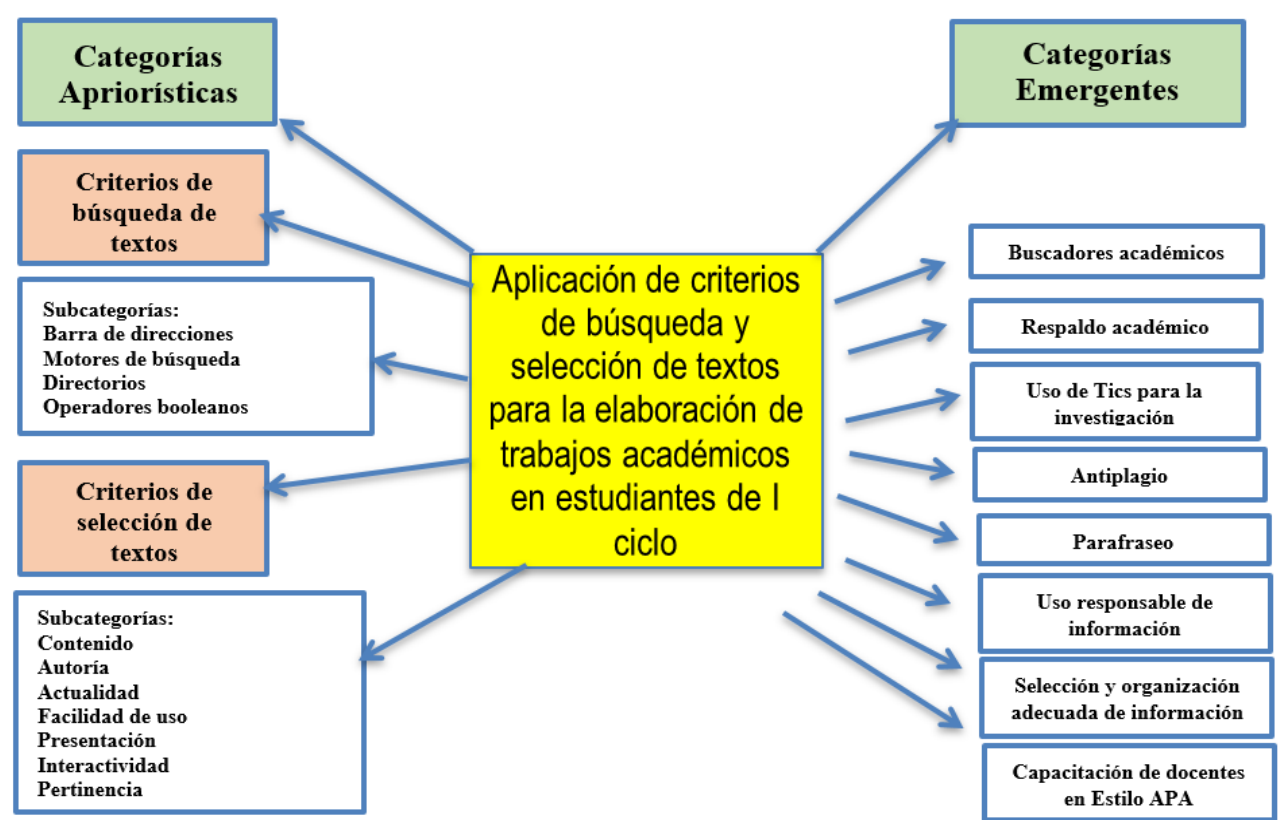

Figura 1. Presentación de las categorías apriorísticas y las categorías emergentes. 


\section{Conclusiones}

Como primera conclusión, se ha identificado el desconocimiento masivo de los estudiantes con respecto a los buscadores académicos, los operadores booleanos, la técnica del fichaje, el parafraseo y el uso de las normativas APA. Esto demuestra que los estudiantes de ambas modalidades, presencial y semipresencial (CGT) carecen de estrategias para la búsqueda y selección de información. A pesar de que los estudiantes de CGT (carreras para gente que trabaja), tienen estudios técnicos, lastimosamen-te, no tienen conocimientos amplios de búsqueda de información.

En lo que respecta al diagnóstico sobre los criterios de búsqueda de textos académicos en la modalidad presencial, la segunda conclusión comprende la información que sigue: (a) existe deficiencia en las técnicas de búsqueda de fuentes de información; (b) existe desconocimiento de buscadores académicos adecuados; (c) no hay diferenciación de fuentes primarias de secundarias; (d) no hay buen filtro de información académica, y (e) hay desconocimiento de aplicativos académicos que pueden ser usados en celulares smartphone o iPhone. A través de los talleres, se logró solucionar gran parte del problema. Los puntos más resaltantes fueron los siguientes: los estudiantes tienen las ventajas para aprender a usar todo lo que sea virtual, solo hay que ser pacientes y elaborar momentos exclusivos para enseñar estas herramientas y, habiendo aprendido sobre el uso de los buscadores académicos, el tesauro UNESCO y sobre los operadores booleanos, se despierta la curiosidad de los participantes y empiezan a investigar sobre los diversos recursos virtuales para mejorar en el ámbito de la investigación. Además, lo comparten en clase entre sus compañeros e, incluso, solicitan un espacio de la sesión al docente para comentar sobre ello.

En síntesis, con respecto a la modalidad presencial, se ha registrado en esta investigación categorías emergentes producto de los hallazgos. Estas responden a las necesi-dades más urgentes de los estudiantes de primer ciclo con respecto a los criterios de búsqueda de textos académicos. Dichas categorías son las que siguen: (a) buscadores académicos, (b) uso de las TICs para la investigación y (c) identificación de textos científicos.

Por otro lado, en lo que respecta al diagnóstico sobre los criterios de búsqueda de textos académicos en la modalidad semipresencial (CGT), se halló lo siguiente: (a) deficiencias al aplicar criterios de búsqueda y selección de fuentes, (b) falta de familia-ridad con la actividad académicas y constantes pérdidas de clases por feriados que generaban retrasos y (c) desactualización de práctica de estrategias. Con respecto a CGT, se encontraron en los hallazgos más aspectos de mejora. Así, los estudiantes participantes no tienen familiaridad con el uso de recursos tecnológicos y necesitan de apoyo y guía constante. Asimismo, los alumnos que tenían conocimientos tecnológicos ayudaban a los compañeros del equipo, lo cual denota gran capacidad de apoyo cola-borativo. De los aspectos más destacados de este trabajo, sobre todo con este módulo II del curso, se tiene la continua ausencia de estudiantes por diversos motivos, la mayoría de índole laboral y familiar, que ha afectado el trabajo disparejo de los talleres en las sesiones presenciales. Solo aquellos que estuvieron presentes en los talleres identifica-ron mejorías en estrategias de búsqueda a través del conocimiento de otros repositorios más allá de Google. De esta forma, a través de los talleres propuestos, se pudo obtener resultados interesantes con respecto a la adecuada aplicación de criterios de búsqueda de textos académicos.

En síntesis, con respecto a la modalidad semipresencial (CGT), se ha registrado en esta investigación categorías emergentes producto de los hallazgos. Con ello, se res-ponde a las necesidades más urgentes de los estudiantes de primer ciclo con respecto a los criterios de búsqueda de textos académicos. Dichas categorías son las que siguen: (a) necesidad de un respaldo académico y (b) criterios para identificación de in-formación confiable a través de los buscadores académicos. 
La tercera conclusión se refiere al diagnóstico sobre los criterios de selección de textos en la modalidad presencial. Se halló lo siguiente: (a) bajo nivel en redacción y exceso de errores ortográficos, y (c) evidencia de plagio en pleno proceso de investigación. Al respecto, a través de los talleres, se logró solucionar gran parte del problema. Así, si bien el uso de la tecnología hace práctico el trabajo de investigación, es oportuno volver a lo tradicional para valorar las herramientas de investigación. Cuando los jóvenes usaban las fichas manuales, se percataban de ciertos detalles que no suelen ser notados cuando se elabora de manera virtual (la trascripción ayuda a comprender mejor el contenido de la fuente de información consultada). Asimismo, tanto el uso de fichas manuales como virtuales ayudó a seleccionar la información necesaria para su investigación. Su esquema de redacción en pleno desarrollo fue de ayuda para la clasificación de las fuentes de información. De esta forma, a través de los talleres propuestos, se pudo obtener resultados interesantes con respecto a la adecuada aplicación de criterios de selección de textos académicos.

En síntesis, con respecto a la modalidad presencial, se ha registrado en esta investigación categorías emergentes producto de los hallazgos, las que responden a las necesidades más urgentes de los estudiantes de primer ciclo con respecto a los criterios de selección de textos académicos. Estas son las siguientes: (a) estrategias de antiplagio, a través de uso adecuado del parafraseo; (b) uso responsable de información; (c) selección adecuada de información y (d) importancia de capacitación de docentes en estilo APA.

En lo que respecta al diagnóstico sobre los criterios de selección de textos en la modalidad semipresencial (CGT), se halló lo siguiente: (a) nivel medio de redacción, (b) deficiencia en la selección y organización de información, (c) confusión a la hora de exponer sus ideas, (d) posibilidad de plagio. En relación con ello, a través de los talleres, se logró identificar los siguientes aspectos: (a) la importancia del uso de fichas manuales y no digitales, (b) el desconocimiento del uso del léxico académico y (c) el necesa-rio apoyo de un estilo de redacción (en este caso, del estilo APA). Al respecto, el ha-llazgo más resaltante ha sido la disposición y apertura de aprendizaje por parte de los estudiantes durante los talleres. A partir de ello, muchos estudiantes precisaron la necesidad de un seguimiento permanente. Por otro lado, la interacción con un especialista en APA ha facilitado la comunicación, y las dudas muy específicas con respecto al uso de redacción y aprendizajes de citas directas e indirectas. Se evidenció que algunos estudiantes (3) denotaban conocimientos autodidactas del uso de APA y que los talleres les ayudaron a reforzar la constatación del saber filtrar los tipos de documentos apro-piados para los trabajos de investigación. De esta forma, a través de los talleres pro-puestos, se pudo obtener resultados interesantes con respecto a la adecuada aplicación de criterios de selección de textos académicos.

En síntesis, con respecto a la modalidad semipresencial (CGT), se ha registrado en esta investigación categorías emergentes producto de los hallazgos. Estas responden a las necesidades más urgentes de los estudiantes de primer ciclo con respecto a los criterios de selección de textos académicos. Las categorías emergentes son las que siguen: (a) organización de la información, (b) utilidad del APA y (c) uso de estrategias de fichaje.

Financiamiento: El presente trabajo ha sido financiado gracias a la convocatoria de investigación aca-démica 2018 de la Universidad Tecnológica de Perú, siendo uno de los proyectos ga-nadores, titulado "La lectoescritura y su contribución en el desarrollo de trabajos aca-démicos en estudiantes del curso de Investigación Académica de la UTP”, ejecutado en el 2019 y finalizado a inicios del 2020. 


\section{Referencias}

Carlino, P. (2003). Leer textos científicos y académicos en la educación superior: obs-táculos y bienvenidas a una cultura nueva. Uni-Pluri/Versidad, 3(2) 17-23. https://aprendeenlinea.udea.edu.co/revistas/index.php/unip/article/viewFile/12289/1114 6

Hernández, R. \& Mendoza, C. (2018). Metodología de la investigación. Las rutas cuantitativa, cualitativa y mixta. México D.F., México: McGraw Hill México.

Hernández, R., Fernández, C., \& Baptista, P. (2014). Metodología de la investigación (6.a ed.). México D.F., México: McGraw Hill.

Moreno, T. (2012). La evaluación de competencias en educación. Sinéctica, 39. https://sinectica.iteso.mx/index.php/SINECTICA/article/view/84/76

Negrete, M. (2002). Criterios de selección para recursos digitales. Scire: representación y $\begin{array}{llll}\text { organización del conocimiento, } & \text { 8(2), }\end{array}$ https://ibersid.eu/ojs/index.php/scire/article/view/1171

Perdomo, B. (2008). Búsqueda y selección de textos en la internet para investigación científica. SABER. Revista Multidisciplinaria del Consejo de Investigación de la Universidad de Oriente, 20 (1), 109-116. https://www.redalyc.org/pdf/4277/427739437016.pdf

Pimienta, J. \& De La Orden, A. (2017). Metodología de la Investigación. México D. F., México: Pearson Educación.

Ricoy, C. (2006). Contribución sobre los paradigmas de investigación. Educação. Revista do Centro de Educação, 31(1), 11-22.

Taylor, S. \& Bogdan, R. (1987). Introducción a los métodos cualitativos de investigación (Vol. 1). Barcelona, España: Paidós. 